\title{
COSMOLOGICAL CONSTRAINTS ON NEUTRINO MASSES AND MIXINGS.
}

\author{
A. D. DOLGOV \\ INFN, sezione di Ferrara, Via Paradiso, 12 - 44100 Ferrara, Italy \\ and \\ ITEP, Bol. Cheremushkinskaya 25, Moscow 113259, Russia \\ E-mail: dolgov@fe.infn.it
}

\begin{abstract}
The bounds on neutrino masses and mixing that follows from the data on light element abundances, large scale structure formation, and angular fluctuations of cosmic microwave background radiation are analyzed. The role of neutrino oscillations in BBN and the bounds on cosmological lepton asymmetry are discussed.
\end{abstract}

\section{Introduction}

Neutrinos have the weakest interactions among all known elementary particles. They have also the smallest mass among all known massive particles. These two properties, on one hand, make it difficult to study neutrino properties directly, in particular, to measure their mass in laboratories. On the other hand, the same properties make neutrinos very important cosmologically and, at the present time, measuring neutrino masses looking at the sky seems more promising than terrestrial experiments. Significant cosmological role played by neutrinos arises from their large number density. Neutrinos are the second most abundant particles in the universe, after photons in Cosmic Microwave Background Radiation (CMBR) with the number density $n_{\gamma} \approx 410 / \mathrm{cm}^{3}$. According to the standard cosmology, the universe is filled, in addition to CMBR, by the Cosmic Neutrino Background Radiation $(\mathrm{C} \nu \mathrm{BR})$ with the present-day number density:

$$
n_{\nu_{a}}^{(0)}=n_{\bar{\nu}_{a}}^{(0)} \approx 56 / \mathrm{cm}^{3}
$$

for any neutrino flavor $a=e, \mu, \tau$. It is usually (but not always) assumed that neutrinos are not degenerate (i.e. their chemical potentials are zero or negligibly small) and the number densities of neutrinos and antineutrinos are equal. 
However, contrary to well observed CMBR, the existence of $\mathrm{C} \nu \mathrm{BR}$ is only a theoretical prediction, and though practically nobody has any doubts of that, direct observation of $\mathrm{C} \nu \mathrm{BR}$ is still missing and it seems that there is no chance for direct registration of cosmic neutrinos today and maybe even in the foreseeable future. Thus, one has to rely on indirect methods studying features imprinted by cosmic neutrinos on:

(1) formation and evolution of astronomical Large Scale Structure (LSS);

(2) angular fluctuations of CMBR;

(3) light element abundances created at Big Bang Nucleosynthesis $(\mathrm{BBN})$

(4) propagation of Ultra High Energy Cosmic Rays (UHECR).

In what follows we will consider the first three subjects only. One can find a more detailed discussion of the issues presented below, as well as of the problem of interaction of UHECR with $\mathrm{C} \nu \mathrm{BR}$, and hopefully a complete list of references in the review ${ }^{1}$.

\section{Early History of Neutrinos.}

When the temperature of the cosmic plasma was above a few $\mathrm{MeV}$, neutrinos were in thermal equilibrium with the electromagnetic component of the plasma, i.e. with photons and $e^{+} e^{-}$-pairs. Electronic neutrinos decoupled from $e^{+} e^{-}$-pairs when the temperature dropped below $T_{d}^{(e)} \approx 1.9 \mathrm{MeV}$, while $\nu_{\mu}$ and $\nu_{\tau}$ decoupled a little earlier at $T_{d}^{(\mu)} \approx 3.1 \mathrm{MeV}$. At the moment of decoupling and later down to $T \approx m_{e}=0.511 \mathrm{MeV}$ temperatures of neutrino and electromagnetic components were equal, $T_{\nu}=T_{\gamma}$. Below $T=m_{e}$ the annihilation of $e^{+} e^{-}$-pairs heats up photons and themselves, while leaves neutrino temperature intact. As a result the initial equilibrium ratio of neutrino-to-photon number densities becomes diluted by the factor 4/11:

$$
\frac{n_{\nu}+n_{\bar{\nu}}}{n_{\gamma}}=\frac{3}{4} \rightarrow \frac{3}{11}
$$

From this result the present-day neutrino number density (1) is obtained. Any additional energy release after $T_{d}^{(a)}$ which might increase the photon number density would correspondingly diminish $n_{\nu}^{(0)}$.

Neutrino spectrum is close to the equilibrium form:

$$
f_{\nu}^{(e q)}=\left[\exp \left(p / T_{\nu}-\xi\right)+1\right]^{-1}
$$


where $p$ is the neutrino momentum, $T_{\nu}$ is the temperature, and $\xi=\mu / T$ is dimensionless chemical potential; for adiabatic expansion $\xi$ remains constant. Usually chemical potentials of different neutrino species are assumed to be negligible, at the level of baryonic asymmetry, $10^{-9}$, though much larger values, even close to 1 , are not excluded. Moreover, there exist theoretical models which predict a small baryon asymmetry and simultaneously large lepton ones. Equilibrium with respect to $\bar{\nu} \nu$-annihilation enforces $\xi_{\nu}+\xi_{\bar{\nu}}=0$ but if neutrino charge asymmetry is generated at low temperatures this condition may be violated.

One more comment is in order: if neutrino mass is non-negligible in comparison with the temperature, the spectrum (3) is non-equilibrium because the latter contains $\exp (E / T)$ but not $\exp (p / T)$. At the present time $T_{\nu} \approx 0.714 T_{\gamma} \approx 1.68 \cdot 10^{-4} \mathrm{eV}$. And if the neutrino mass is larger than this value the deviations from the usual Fermi distribution may be significant. This must be true at least for two out of three neutrinos because from the atmospheric neutrino anomaly $\delta m^{2}=(2-5) \cdot 10^{-3} \mathrm{eV}^{2}$ and from the solar neutrino deficit and KAMLAND data $\delta m^{2}=(6-7) \cdot 10^{-5} \mathrm{eV}^{2}$.

However, even at high temperatures $\left(T \gg m_{\nu}\right)$ a deviation from equilibrium was non-negligible. Indeed, the decoupling of neutrinos from $e^{+} e^{-}$pairs is not instantaneous and the annihilation $e^{+} e^{-} \rightarrow \nu \bar{\nu}$ at $T \leq m_{e}$ would heat the neutrino component of the plasma and distort its spectrum. According to analytical estimates of ref. ${ }^{2}$ the spectral distortion for $\nu_{e}$ has the form:

$$
\frac{\delta f_{\nu_{e}}}{f_{\nu_{e}}} \approx 3 \cdot 10^{-4} \frac{E}{T}\left(\frac{11}{4} \frac{E}{T}-3\right)
$$

Most accurate numerical solution of the kinetic equation that governs nonequilibrium corrections to the neutrino spectrum was performed in ref. ${ }^{3}$. According to the calculations the excess of energy density of $\nu_{e}$ and $\nu_{\mu, \tau}$ are respectively $\delta \rho_{\nu_{e}} / \rho_{\nu}=0.9 \%$ and $\delta \rho_{\nu_{\mu}, \nu_{\tau}} / \rho_{\nu}=0.4 \%$. Together with the plasma corrections ${ }^{4}$ which diminish the energy density of the electromagnetic component, the total relative rise of neutrino energy density reaches approximately $4 \%$. This phenomenon has very little impact on production of primordial ${ }^{4} \mathrm{He}$ but can be observable in the shape of the angular fluctuations of CMBR in the forthcoming Planck mission. If observed, then together with BBN, it would present evidence of physical processes which took place in the universe when she was about 1 sec old. The corresponding red-shift is about $10^{10}$. More details and references relevant to the subject of this section can be found in the review ${ }^{1}$. 


\section{Gerstein-Zeldovich and LSS Bounds on Neutrino Mass}

Knowing the present-day number density of relic neutrinos one can easily calculate their energy density and obtain an upper limit on their mass. Such bound was derived in 1966 by Gerstein and Zeldovich ${ }^{5}$ The result was re-derived 6 years later by Cowsic and Mc Lelland ${ }^{6}$ but in their work the effect was overestimated by the factor $22 / 3$. In contemporary form the limits reads:

$$
\sum_{a} m_{\nu_{a}} \leq 94 \mathrm{eV} \Omega h^{2}
$$

where the sum is taken over all neutrino species, $a=e, \mu, \tau ; \Omega=\rho / \rho_{c}$ is the cosmological mass fraction of matter, $\rho_{c}=10.5 h^{2} \mathrm{keV} / \mathrm{cm}^{3}$, and $h$ is the dimensionless Hubble parameter, $h=H / 100 \mathrm{~km} / \mathrm{sec} / \mathrm{Mpc} \approx 0.7$.

According to the different and independent pieces of astronomical data $\Omega<0.3$ and correspondingly $\sum_{a} m_{\nu_{a}}<14 \mathrm{eV}$. Since the data on neutrino oscillations ${ }^{7}$ show that the neutrino mass difference is much smaller than $\mathrm{eV}$, the mass of any neutrino flavor should be below $4.7 \mathrm{eV}$.

This limit can be further improved if one takes into account a possible role that massive neutrinos might play in the process of formation of the large scale structure of the universe. The point is that in neutrino dominated universe all the structures on the scales smaller than the neutrino free streaming length, $l_{\nu}$, should be erased. The latter can be estimated as the distance that neutrinos could free-stream in the universe before they became non-relativistic. The mass inside the free-streaming volume can be estimated as $M_{\nu} \approx 5 \cdot 10^{17} M_{\odot}\left(1 \mathrm{eV} / m_{\nu}\right)^{2}$. If density perturbations are common for all particles (this is true for adiabatic perturbations created by inflation), then neutrino out-stream would leave behind less power at small scales inhibiting structure formation at these scales. The larger is $\Omega_{\nu}$ the larger is the effect. Moreover, the larger is the fraction of relativistic (hot) dark matter the later structure formation begins. Hence observation of structures at large red-shifts allows to conclude that $\Omega_{\nu}<0.1$ and $m_{\nu}<1.5 \mathrm{eV}^{8}$. The analysis of the recent data from $2 \mathrm{dF}$ Galaxy Redshift Survey ${ }^{10}$ permitted to put the limit $\sum_{a} m_{\nu_{a}}<2.2 \mathrm{eV}$ or individual masses should be below $0.73 \mathrm{eV}$. As argued in ref. ${ }^{9}$ detailed analysis of structure formation by the Sloan Digital Sky Survey would be sensitive to neutrino mass at the level of $(\mathrm{a}$ few $) \times 0.1 \mathrm{eV}$.

This limits are based on certain assumptions about the form of the spectrum of perturbations, their character (adiabatic or isocurvature), and, what is probably the safest one, about neutrino interactions. A question 
may arise in this connection whether neutrinos can supply all the dark matter in the universe if we relax any or all these assumptions, introducing an arbitrary shape of the perturbation spectrum and/or new neutrino interactions (of course inside the established limits). The answer would still be negative because of the Tremain-Gunn limit ${ }^{11}$. This limit manifests quantum mechanical Fermi exclusion principle at the kiloparsec scales and demands that neutrinos must be heavier than roughly speaking $100 \mathrm{eV}$ if they form all dark matter in galaxies. So it seems that the only way out is to make a crazy assumption that neutrinos are bosons and not fermions which in light of the discussed today search of CPT violation maybe looks not so crazy because CPT-theorem is heavily based on the standard relation between spin and statistics.

\section{Neutrino Mass and CMBR}

The impact of neutrinos on the shape of angular spectrum of CMBR temperature is based on the following two effects (for details see e.g. the recent review $\left.{ }^{12}\right)$. First, a change of the energy density of relativistic matter (neutrinos) would change the cosmological expansion regime and this in turn would change the physical size of the horizon at recombination. It would shift the positions of the acoustic peaks in the temperature fluctuations. This effect is relatively weak. More important is another one which changes the heights of the peaks because an admixture of relativistic matter gives rise to an amplification of acoustic oscillations since relativistic matter creates gravitational force which varies with time creating resonance amplification of the acoustic oscillations. These phenomena permit to measure neutrino mass and the number of neutrino families (or in other words, the energy density of neutrinos) in the recombination epoch.

The recent measurements of the angular fluctuations of CMBR by WMAP ${ }^{13}$, together with the analysis of LSS by $2 \mathrm{dF}$, permitted to impose surprisingly strong upper limit on neutrino mass

$$
\sum_{a} m_{\nu_{a}}<0.69 \mathrm{eV}
$$

that is for mass degenerate neutrinos $m_{\nu}<0.23 \mathrm{eV}$ at $95 \% \mathrm{CL}$; see also the discussion of this result and of the role of primers in ref. ${ }^{14}$.

Immediately after publication of the first WMAP data, there appeared several papers ${ }^{15}$ where the number of neutrino families were evaluated. Their results are, roughly speaking, $N_{\nu}=1-7$ depending upon the analysis 
and priors. Anyhow $N_{\nu}>0$ and thus CMBR confirms, independently on $\mathrm{BBN}$, that $\mathrm{C} \nu \mathrm{BR}$ (or some other relativistic background) indeed exists.

The accuracy of the data is not good enough to compete with the determination of $N_{\nu}$ from BBN (see sec. 5) to say nothing about a registration of $4 \%$ addition to the neutrino energy discussed in sec. 2. However the forthcoming Planck mission may be sensitive to this effect and overtake BBN in determination of $N_{\nu}$. One should keep in mind that the impact of neutrino energy on BBN and CMBR may be different depending upon the form of the spectral distortion of electronic neutrinos $f_{\nu_{e}}(E)$. The effect, which was calculated for massless neutrinos, also depends upon the value of neutrino mass. This may be essential at the recombination epoch.

\section{Neutrinos and BBN}

Abundances of light elements $\left({ }^{2} \mathrm{H},{ }^{3} \mathrm{He},{ }^{4} \mathrm{He}\right.$, and $\left.{ }^{7} \mathrm{Li}\right)$ produced at $\mathrm{BBN}$ at $T=1-0.07 \mathrm{MeV}$ ( $\mathrm{t}=1-200 \mathrm{sec})$ depend upon the following quantities:

(1) Number density of baryons, $\eta_{10}=10^{10} n_{B} / n_{\gamma}$. In the previous century the value of this parameter was determined from BBN itself through comparison of the predicted deuterium abundance with observations. Now after measurements of CMBR angular fluctuations by MAXIMA, BOOMERANG and DASI ${ }^{16}$ and confirmed by WMAP ${ }^{13}$ this parameter is independently fixed at $\eta_{10}=6 \pm 0.3$.

(2) Weak interaction rate which is expressed through the neutron lifetime, now well established, $885.7 \pm 0.8 \mathrm{sec}^{17}$.

(3) Cosmological energy density at the period of BBN. The latter is usually parametrized as the effective number of additional neutrino species $\Delta N_{\nu}=N_{\nu}-3$. This type of parametrization is flawless for relativistic energy, while for another form of energy (e.g. nonrelativistic or vacuum-like) its effect on the production of different light elements may deviate from that induced by neutrinos.

(4) Neutrino degeneracy, i.e. a possible non-vanishing values of neutrino chemical potentials $\xi_{a}$. While for $\nu_{\mu}$ and $\nu_{\tau}$ non-zero $\xi_{\mu, \tau}$ are equivalent to an increase of $N_{\nu}$, degeneracy of electronic neutrinos has a much stronger impact on BBN because the frozen neutron-toproton ratio is exponentially sensitive to the magnitude (and sign) of chemical potential of electronic neutrinos, $n_{n} / n_{p} \sim \exp \left(-\xi_{\nu_{e}}\right)$.

(5) Energy spectrum of $\nu_{e}$. If the spectrum is distorted then the equilibrium of reactions $n \nu_{e} \leftrightarrow p e^{-}$and $n e^{+} \leftrightarrow p \overline{\nu_{e}}$ is shifted and could result either in an increase or decrease of $n_{n} / n_{p}$ depending upon 
the form of distortion.

The upper bound on the effective number of neutrino families, found from BBN, had a rather strong time evolution and it changed from $\Delta N_{\nu}=3-5$ in the original papers ${ }^{18}$ down to 0.3 and even to 0.1 in subsequent literature. The existing data on the light element abundances is still controversial ${ }^{19}$ and possibly the situation described in ref. ${ }^{20}$ still remains true, which is roughly the following $n_{\nu}=3 \pm 0.5$.

The most accurate upper bound on chemical potential of a single neutrino species under the conservative assumptions that $\Delta N<1$ and other chemical potentials are vanishingly small was obtained in ref. ${ }^{21}$ and reads: $\left|\xi_{\nu_{\mu}, \nu_{\tau}}\right|<1.5$ and $\left|\xi_{\nu_{e}}\right|<0.1$. This bound would be relaxed if a conspiracy between different chemical potentials is allowed such that the effect of a large $\left|\xi_{\nu_{\mu}, \nu_{\tau}}\right|$ is compensated by $\left|\xi_{\nu_{e}}\right|$ (or vice versa). The best limit is presented in ref. ${ }^{22}$ where the combined inputs from BBN and CMBR have been used: $\left|\xi_{\nu_{\mu}, \nu_{\tau}}\right|<2.6$ and $\left|\xi_{\nu_{e}}\right|<0.2$.

The bounds quoted above are valid if neutrinos are not mixed. Otherwise, charge asymmetry of a certain neutrino flavor would be redistributed between all neutrino species and the bound would be determined by the most sensitive asymmetry of $\nu_{e}$. This problem was analyzed in ref. ${ }^{23}$ numerically and analytically in ref. ${ }^{1}$, where it was shown that for Large Mixing Angle solution of the solar neutrino deficit the transformation is quite efficient and equilibration of all chemical potentials by oscillations is achieved. This leads to the common bound for all neutrino chemical potentials

$$
\left|\xi_{a}\right|<0.07
$$

Similar investigation both analytical and numerical was also performed in the papers ${ }^{24}$.

If a new sterile neutrino (or several sterile species) mixed with active ones exists, the impact of neutrino oscillation on BBN would be more interesting than in the case of active-active mixing. The $\nu_{s} \leftrightarrow \nu_{a^{-}}$ transformations would excite additional neutrino species leading to an increase of the effective number of neutrinos, $\Delta N>0{ }^{25,26}$, could distort the spectrum of $\nu_{e}{ }^{26,27}$ and could generate a large lepton asymmetry in the sector of active neutrinos by MSW-resonance ${ }^{28}$. (More references and discussion can be found in ${ }^{1}$.)

In the non-resonance case, i.e. for $m_{\nu_{s}}>m_{\nu_{a}}$ it is rather easy to estimate the production rate of sterile neutrinos in the early universe through 
oscillations and to obtain the following bounds on the oscillation parameters ${ }^{29}$ :

$$
\begin{aligned}
& \left(\delta m_{\nu_{e} \nu_{s}}^{2} / \mathrm{eV}^{2}\right) \sin ^{4} 2 \theta_{\text {vac }}^{\nu_{e} \nu_{s}}=3.16 \cdot 10^{-5}\left[\log \left(1-\Delta N_{\nu}\right)\right]^{2} \\
& \left(\delta m_{\nu_{\mu} \nu_{s}}^{2} / \mathrm{eV}^{2}\right) \sin ^{4} 2 \theta_{\text {vac }}^{\nu_{\mu} \nu_{s}}=1.74 \cdot 10^{-5}\left[\log \left(1-\Delta N_{\nu}\right)\right]^{2}
\end{aligned}
$$

In this result a possible deficit of $\nu_{e}$ created by the transformation of the latter into $\nu_{s}$, when refilling of $\nu_{e}$ by $e^{+} e^{-}$-annihilation is already weak, is not taken into account. This effect would strengthen the bound.

Numerical solution of kinetic equations governing neutrino oscillations in the early universe was performed in ref. ${ }^{30}$ under assumption of kinetic equilibrium, so the neutrinos are described by a single momentum state with the thermally average value of the energy $E=3.15 T$. For the non-resonant case the obtained results are somewhat stronger than those presented above, $(8,9)$. For the resonant case it is questionable if thermal averaging is a good approximation because the position of resonance depends upon the neutrino momentum. One more complication is that now is known that all active neutrinos are strongly mixed and their mutual transformation should be taken into account together with $\left(\nu_{a}-\nu_{s}\right)$-oscillations ${ }^{31}$. The active neutrino mixing noticeably changes the previously obtained cosmological limits for mixing with $\nu_{s}$. Moreover, the solution of momentum dependent kinetic equations shows that kinetic equilibrium is strongly broken (at least for some values of the oscillation parameters) and, in particular, the spectrum of $\nu_{e}$ is distorted leading to a shift of the $n / p$-ratio for interesting values of mass difference between $\nu_{s}$ and active neutrinos. The calculations are complicated by the appearance of one, two or even three resonances if sterile neutrino is lighter than one, two or all three active ones. Anyhow in the resonance case the cosmological bounds on the mixing between sterile and active neutrinos are considerably stronger than those in non-resonance case. Thus if a large mixing to $\nu_{s}$ is discovered it would mean that the lepton asymmetry of the universe is non-negligible ${ }^{31}$, because the latter might "cure" the effect of $\nu_{a}-\nu_{s}$ oscillations on BBN.

\section{Conclusion}

Thus we see that cosmology is becoming sensitive to the values of neutrino masses approaching $\sqrt{\delta m^{2}}$. So one may hope that neutrino will be the first particle whose mass will be measured by astronomers by the combined data from CMBR and LSS.

The number of additional neutrinos at BBN is limited by 0.5 (though 1 
is probably still not excluded) with a possibility to improve this limit down to 0.1 .

The observed in experiment strong mixing between active neutrinos allows cosmological lepton asymmetry to be relatively low, smaller than 0.1. This excludes, in particular, cosmological models where large chemical potential of neutrinos might be essential for large scale structure formation.

A possible mixing between active and sterile neutrinos is restricted by BBN at much stronger level than by direct experiment.

Planck mission may be sensitive to additional contribution to neutrino energy density at per cent level and thus will be able to trace physical processes in the universe at red shift of $10^{10}$.

I am grateful to F. Villante for critical comments.

\section{References}

1. A.D. Dolgov, Phys. Repts. 370, 333 (2002).

2. A.D. Dolgov and M. Fukugita, JETP Lett. 56 (1992) 123 [Pisma Zh. Eksp. Teor. Fiz. 56 (1992) 129];

A.D. Dolgov and M. Fukugita, Phys. Rev. D46, 5378 (1992).

3. A.D. Dolgov, S.H. Hansen and D.V. Semikoz, Nucl. Phys. B503 (1997) 426; Nucl. Phys. B543 (1999) 269.

4. A.F. Heckler, Phys. Rev. D49 , 611 (1994);

R.E. Lopez, S. Dodelson, A. Heckler and M.S. Turner, Phys. Rev. Lett. 82, 3952 (1999).

5. S.S. Gerstein and Ya.B. Zeldovich, Pis'ma ZhETF, 4, 174 (1966) [English translation JETP Letters 4, 120 (1966)].

6. R. Cowsik and J. McClelland, Phys. Rev. Lett. 29, 669 (1972).

7. See e.g. the talks by E. Lisi, T. Schwetz, A.Y. Smirnov at this Conference.

8. R.A.C. Croft, W. Hu, and R. Davé, Phys. Rev. Lett. 83, 1092 (1999);

W. Hu, D.J. Eisenstein and M. Tegmark, Phys. Rev. Lett. 80, 5255 (1998);

M. Fukugita, G.-C. Liu, N. Sugiyama, Phys. Rev. Lett. 84, 1082 (2000);

A.R. Cooray, Astron. Astrophys. 348, 31 (1999);

E. Gawiser, Proceedings of PASCOS99 Conference, Lake Tahoe, CA 1999, astro-ph/0005475.

9. W. Hu, D.J. Eisenstein, M. Tegmark and M.J. White, Phys. Rev. D59, 023512 (1999).

10. O. Elgaroy et al, Phys. Rev. Lett. 89, 061301 (2002).

11. S. Tremaine and J.E. Gunn, Phys. Rev. Lett. 42, 407 (1979).

12. W. Hu and S. Dodelson, Annu. Rev. Astron. and Astrophys. 40, 171 (2002).

13. D.N. Spergel et al, astro-ph/0302209.

14. O. Elgaroy and O. Lahav, JCAP 0304, 004 (2003).

15. P. Crotty, J. Lesgourgues and S. Pastor, astro-ph/0302337;

S. Hannestad, astro-ph/0303076;

E. Pierpaoli, astro-ph/0302465; 
V. Barger, J.P. Kneller, H.-S. Lee, D. Marfatia and G. Steigman, hep$\mathrm{ph} / 0305075$.

16. A.T. Lee et al, Astrophys. J. 561, L1 (2001);

C.B. Netterfield et al, Astrophys. J. 571 (2002) 604;

N.W. Halverson et al, Astrophys. J. 568 (2002) 38.

17. K. Hagiwara et al, Phys. Rev. D66,010001 (2002).

18. V. F. Shvartsman, Pisma Zh. Eksp. Teor. Fiz. 9, 315 (1969) [JETP Lett. 9, 184 (1969)];

G. Steigman, D.N. Schramm and J.R. Gunn, Phys. Lett. B66, 202 (1977).

19. A.D. Dolgov, Nucl. Phys., Proc. Suppl. B110, 137 (2002).

20. E. Lisi, S. Sarkar and F.L. Villante, Phys. Rev. D59, 123520 (1999);

G. Fiorentini, E. Lisi, S. Sarkar and F.L. Villante, Phys. Rev. D58, 063506 (1998).

21. K. Kohri, M. Kawasaki and K. Sato, Astrophys. J. 490, 72 (1997).

22. S.H. Hansen, G. Mangano, A. Melchiorri, G. Miele and O. Pisanti, Phys. Rev. D65, 023511 (2002).

23. A.D. Dolgov, S.H. Hansen, S. Pastor, S.T. Petcov, G.G. Raffelt and D.V. Semikoz, Nucl.Phys. B632 363 (2002).

24. C.Lunardini and A.Yu.Smirnov, Phys. Rev. D64, 073006 (2001); Y.Y.Y. Wong, Phys. Rev. D66 025015 (2002); K.N. Abazajian, J.F. Beacom and N.F. Bell, Phys.Rev. D66 013008 (2002).

25. A.D.Dolgov, Yad. Fiz. 33, 1309 (1981); [English translation: Sov. J. Nucl. Phys. 33, 700 (1981).

26. R. Barbieri and A.D. Dolgov, Phys. Lett. B237, 440 (1990); K. Kainulainen, Phys. Lett. B244, 191 (1990).

27. D.P. Kirilova and M.V. Chizhov, Phys. Lett. B393, 375 (1997).

28. R. Foot, M.J. Thomson and R.R. Volkas, Phys. Rev. D53, 5349 (1996).

29. A.D. Dolgov, Surveys in High Energy Physics, 17, 91 (2002) (lectures presented at ITEP Winter School, February, 2002).

30. K. Enqvist, K. Kainulainen and M. Thomson, Nucl. Phys. B373, 498 (1992).

31. A.D. Dolgov and F.L. Villante, work in progress. 